\title{
Solvability of Convolution Operators
}

\author{
By \\ Yasunori OKADA*
}

\section{Contents}

$\S 0$. Introduction

$\S 1$. Preliminaries

1.1. The sheaf $Q$ of Fourier hyperfunctions

1.2. Topologies

1.3. Fourier transformation and convolution

1.4. Ultradistributions

1.5. Kawai's abstract sufficient condition (S)

$\S 2$. Surjectivity of convolution operators with ultradistribution kernel on the space of hyperfunctions

2.1. Statement of the theorem on the space of hyperfunctions

2.2. One dimensional case

2.3. General case

$\S 3$. Surjectivity of convolution operators on the space of Fourier hyperfunctions

3.1. Statement of the theorems on the space of Fourier hyperfunctions

3.2. Proof of the theorems

References

\begin{abstract}
We study the surjectivity of convolution operators on the spaces of hyperfunctions and Fourier hyperfunctions. On the space of hyperfunctions, we give a sufficient condition (the kernel is a nonzero ultradistribution), weaker than earlier conditions. On the space of Fourier hyperfunction, we give a new sufficient condition and new necessary conditions for the surjectivity. Especially in one dimensional case, they become a sufficient and necessary condition. To this aim we use the Fourier analysis as in L. Ehrenpreis [E-2] and T. Kawai [Ka-1].
\end{abstract}

Communicated by T. Kawai, November 16, 1992, Revised April 26, 1993.

1991 Mathematics Subject Classification : 42A85, 46F15.

* College of Arts and Sciences, Chiba University, 1-33 Yayoi-cho Inage-ku, Chiba 263 Japan. 


\section{$\S 0$. Introduction}

Let $P(D)$ be a linear differential operator of finite order with constant coefficients. If we consider $P(D)$ as an operator on the space $\mathscr{B}\left(\boldsymbol{R}^{n}\right)$ of hyperfunctions, the operation of $P$ on $f \in \mathscr{B}\left(\mathbb{R}^{n}\right)$ can be rewritten by using convolution as

$$
P f=(P(D) \delta) * f .
$$

Here $\delta$ is Dirac's delta function. Thus we may regard the operator $P$ as a convolution operator $\mu *$ whose kernel $\mu$ is a distribution supported in the origin. The operator $\mu *$ makes sense not only the case where $\mu$ is a distribution with compact support but also a compactly supported hyperfunction. Moreover the operator $\mu *$ can be considered as an operator on the space $Q\left(D^{n}\right)$ of Fourier hyperfunctions. (Refer to $\S 1.1$ for their definitions.)

When $\mu *$ is a differential operator of finite order with constant coefficients, $\mu *$ is surjective on $\mathscr{B}\left(\mathbb{R}^{n}\right)$. (See Harvey [H].) We will study in this paper the surjectivity of $\mu *$ in a general situation.

For the surjectivity on $\mathscr{B}\left(\mathbb{R}^{n}\right)$, the following two sufficient conditions are already known in Martineau [M] and Kawai [Ka-1]:

1) The support of $\mu$ is one point,

2) $\mu$ is a non-zero compactly supported distribution.

The both conditions have been shown to be sufficient by reducing to Kawai's abstract condition (S). (Refer to $\S 1.5$.)

The aim of this paper is to give conditions on the kernel $\mu$ for the convolution operators $\mu *$ to be surjective on the spaces of hyperfunctions and Fourier hyperfunctions.

First in the second chapter, we will consider the problem on $\mathscr{B}\left(\boldsymbol{R}^{n}\right)$ and show a generalization of the sufficient condition 2):

$2)^{\prime} \quad \mu$ is a non-zero ultradistribution

to be sufficient. We also reduce this to the condition (S). (Refer to $\S 2.2$ and $\S 2.3$.) At the end of the second chapter, we will explain by a similar argument why the condition 1 ) is sufficient.

In the third chapter, we will deal with the problem on $Q\left(\boldsymbol{D}^{n}\right)$ and give a sufficient condition ( $\left.S^{\prime}\right)$ and $\left(Z^{\prime}\right)$ for the surjectivity. (See Theorem 3.3. The condition (S') is due to Kawai [Ka-1].) Moreover we will show the following:

1) ( $S^{\prime}$ ) is a necessary condition for surjectivity, (see Theorem 3.2.)

2) In the case $n=1,\left(Z^{\prime}\right)$ is also necessary, (see Theorem 3.1.)

3) Two conditions ( $\left.\mathrm{S}^{\prime}\right)$ and ( $Z^{\prime}$ ) are independent. (see Theorem 3.4.)

\section{Acknowledgement}

I would like to express my sincere gratitude to Prof. T. Oshima for guidance and encouragement. 


\section{$\S 1 . \quad$ Preliminaries}

\subsection{The sheaf $Q$ of Fourier hyperfunctions}

We review in this section and the following ones some definitions and basic properties concerned with Fourier hyperfunctions ([Ka-1], $[\mathrm{S}]$ ) and ultradistributions ([Ro-1, 2], [Be], [Bj], [Ko-2, 3]).

We denote by $\boldsymbol{R}^{n}$ (resp. $\boldsymbol{C}^{n}$ ) an $n$-dimensional real (resp. complex) Euclidean space, and define the sphere at infinity by

$$
S_{\infty}^{n-1}=\left(\mathbb{R}^{n} \backslash\{0\}\right) / \sim .
$$

Here $\sim$ is an equivalence relation in $\mathbb{R}^{n} \backslash\{0\}$ given by

$$
x \sim x^{\prime} \Longleftrightarrow k x=x^{\prime} \quad \text { for some } k>0 \text {. }
$$

The equivalence class of $x \in \mathbb{R}^{n} \backslash\{0\}$ is denoted by $x \infty$. We put $\boldsymbol{D}^{n}=\boldsymbol{R}^{n} \sqcup S_{\infty}^{n-1}$ and introduce the induced topology by the map

$$
\widetilde{\sigma}: \boldsymbol{D}^{n} \longrightarrow \boldsymbol{R}^{n}
$$

defined as

$$
\begin{aligned}
\varpi(x) & =\frac{x}{|x|+1} \quad\left(x \in \mathbb{R}^{n}\right), \\
\widetilde{\sigma}(x \infty) & =\frac{x}{|x|} \quad\left(x \infty \in S_{\infty}^{n-1}\right) .
\end{aligned}
$$

We identify $\boldsymbol{C}^{n}=\boldsymbol{R}^{n} \times \sqrt{-1} \boldsymbol{R}^{n}$ with an open subset of $\boldsymbol{D}^{n} \times \sqrt{-1} \boldsymbol{R}^{n}$, and $\boldsymbol{R}^{n}$ with an open subset of $\boldsymbol{D}^{n}$ :

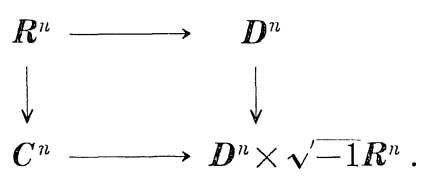

The sheaf of germs of holomorphic functions on $\mathbb{C}^{n}$ is denoted by $\mathcal{O}$ and the one of real analytic functions on $\boldsymbol{R}^{n}$ by $\mathcal{A}$. To define the sheaf of Fourier hyperfunctions, two sheaves $\tilde{\mathcal{O}}$ and $\mathcal{O}$ ot holomorphic functions with growth conditions are defined on $\boldsymbol{D}^{n} \times \sqrt{-1} \boldsymbol{R}^{n}$ in the following way. For an open subset $U$ of $\boldsymbol{D}^{n} \times \sqrt{-1} \boldsymbol{R}^{n}$, the spaces of sections of these sheaves on $U$ are given by

$$
\widetilde{\mathcal{O}}(U)=\left\{f(z) \in \mathcal{O}\left(U \cap \boldsymbol{C}^{n}\right) ; \sup _{z \in K_{\cap} C^{n}}\left|e^{-\varepsilon|\operatorname{Re} z|} f(z)\right|<+\infty\right.
$$

for any compact subset $K$ of $U$ and any positive $\varepsilon$, $\mathcal{Q}(U)=\left\{f(z) \in \mathcal{O}\left(U \cap C^{n}\right)\right.$; for any compact subset $K$ of $U$, theres exists a positive $\delta$ such that $\left.\sup _{z \in K \cap C^{n}}\left|e^{\delta, \operatorname{Re} z \mid} f(z)\right|<+\infty\right\}$. 
Then $\widetilde{\mathcal{O}}$ and $\mathcal{O}$ really become sheaves and satisfy

$$
\left.\widetilde{\mathcal{O}}\right|_{c^{n}}=\left.\mathcal{O}\right|_{c^{n}}=\mathcal{O} \text {. }
$$

Finally recall the definition of space $\mathscr{P}_{*}$ of rapidly decreasing real analytic functions,

$$
\mathscr{P}_{*}=\mathcal{Q}\left(\boldsymbol{D}^{n}\right)=\underset{\boldsymbol{D}^{n} \subset U}{\lim _{\longrightarrow}} \mathcal{Q}(U)
$$

Remark that an element $f$ of $\mathscr{Q}_{*}$ is holomorphic in a tube domain $\mathbb{R}^{n} \times$ $\sqrt{-1}(]-\delta, \delta[)^{n}$ and has an estimate $e^{\varepsilon\left|\operatorname{Re}{ }^{2}\right|}|f(z)|<$ Const. on the above domain with some positive $\delta$ and $\varepsilon$.

Definition 1.1 (The sheaf $Q$ of Fourier hyperfunctions). We define the sheaf $\mathscr{B}$ on $\mathbb{R}^{n}$ of Sato's hyperfunctions and $Q$ on $\boldsymbol{D}^{n}$ of Fourier hyperfunctions by

$$
\begin{aligned}
& \mathscr{B}=\mathcal{H}_{\boldsymbol{R}^{n}}^{n}(\mathcal{O}), \\
& Q=\mathscr{H}_{\boldsymbol{D}^{n}}^{n}(\widetilde{\mathcal{O}})
\end{aligned}
$$

Moreover we set

$$
\mathscr{B}_{c}\left(\boldsymbol{R}^{n}\right)=\left\{\mu \in \mathscr{B}\left(\boldsymbol{R}^{n}\right) ; \quad \operatorname{supp} \mu \text { is compact }\right\} .
$$

We list up several properties of $Q$ :

i) $Q$ is a flabby sheaf on $\boldsymbol{D}^{n}$.

ii) $\left.Q\right|_{R^{n}}=\mathscr{B}$,

iii) An element of $Q\left(\boldsymbol{D}^{n}\right)$ (resp. $\mathscr{B}\left(\boldsymbol{R}^{n}\right)$ ) is expressed as a sum of boundary values of sections of $\widetilde{\mathcal{O}}$. (resp. $\mathcal{O})$.

The above definitions of $\widetilde{\mathcal{O}}, \mathcal{Q}, \mathscr{Q}_{*}$ and $Q$ are due to Kawai.

\subsection{Topologies}

We will use the theory of (FS) and (DFS) spaces which has been developed by Grothendieck [G], S. e Silva [S], Raikov [Ra] and Yoshinaga [Y]. See also Komatsu [Ko-1] for their definitions.

Now let $U$ be an open subset of $\boldsymbol{D}^{n} \times \sqrt{-1} \boldsymbol{R}^{n}$, let $V$ be a relatively compact subset of $U$. If $f$ is holomorphic in $U \cap \boldsymbol{C}^{n}$, then we set for $\boldsymbol{\varepsilon} \in \boldsymbol{R}$

$$
\|f\|_{V, \varepsilon}=\sup _{z \in V \cap C^{n}}\left|e^{-\varepsilon_{1} \operatorname{Re} z_{1}} f(z)\right| .
$$

We define a Banach space $X_{V, \varepsilon}$ by

$$
\begin{aligned}
X_{V, \varepsilon}=\{ & f(z) \in \mathcal{O}\left(V \cap C^{n}\right) ;\|f\|_{V, \varepsilon}<+\infty, \\
& \left.f \text { extends continuously to the closure of } V \cap C^{n} \text { in } C^{n}\right\} .
\end{aligned}
$$

$X_{V, s}$ is endowed with the norm $\|\cdot\|_{V, \varepsilon}$. We introduce the topologies on $\widetilde{\mathcal{O}}(U)$ and $\mathcal{Q}(U)$, and regard them as locally convex spaces by 


$$
\begin{aligned}
& \widetilde{\mathcal{O}}(U)=\lim _{V \in U}{\underset{l}{\varepsilon \downarrow 0}}_{\varepsilon \downarrow} X_{V, \varepsilon}, \\
& \mathcal{Q}(U)=\lim _{V \Xi U} \varliminf_{\varepsilon \downarrow 0} X_{V,-\varepsilon} .
\end{aligned}
$$

We also introduce topologies on $\mathscr{P}_{*}$ and $\mathcal{A}(K)$ by

$$
\begin{aligned}
& \mathcal{P}_{*}=\underline{\lim }_{V \supset \boldsymbol{D}^{n}} \mathcal{Q}(V), \\
& \mathcal{A}(K)=\varliminf_{V \supset K}^{\lim _{\longrightarrow}} \mathcal{O}(V) .
\end{aligned}
$$

Here $K$ is a compact subset of $\boldsymbol{R}^{n}$ and $\mathscr{O}(V)$ is a locally convex space isomorphic to $\widetilde{\mathcal{O}}(V)$. Let $K$ be a compact subset of $\boldsymbol{R}^{n}$, and let $\mathscr{B}[K]$ denote the space of hyperfunctions supported in $K$.

Under the above, $\mathscr{L}_{*}$ and $\mathfrak{A}(K)$ become (DFS) spaces. If we express elements of $Q\left(\boldsymbol{D}^{n}\right)$ and $\mathscr{B}[K]$ as boundary values of holomorphic functions, this makes $Q\left(\boldsymbol{D}^{n}\right)$ and $\mathscr{B}[K]$ (FS) spaces. Moreover we have dualities

$$
\begin{aligned}
& Q\left(\boldsymbol{D}^{n}\right) \longleftrightarrow \mathcal{D}_{*}, \\
& \mathscr{B}[K] \longleftrightarrow \mathcal{A}(K) .
\end{aligned}
$$

\subsection{Fourier transformation and convolution}

First we define Fourier transformation on $\mathscr{P}_{*}$ and $\mathscr{B}_{c}\left(\boldsymbol{R}^{n}\right)$. For $\varphi \in \mathscr{Q}_{*}$, the Fourier transform $\hat{\varphi}$ of $\varphi$ is defined by

$$
\hat{\varphi}(\xi)=\int_{\boldsymbol{R}^{n}} \varphi(x) e^{-\imath x \xi} d x \quad \dot{\xi} \in \boldsymbol{R}^{n} .
$$

Then we have $\hat{\varphi} \in \mathscr{Q}_{*}$. Moreover we have an isomorphism as (DFS) spaces:

$$
\mathscr{F}: \mathscr{Q}_{*} \longrightarrow \mathscr{Q}_{*}: \varphi \longmapsto \hat{\varphi}
$$

For $\mu \in \mathscr{B}_{c}\left(\boldsymbol{R}^{n}\right)$, the Fourier-Laplace transform $\hat{\mu}$ of $\mu$ is defined by

$$
\hat{\mu}(\zeta)=\left\langle\mu, e^{-1 x_{\zeta}}\right\rangle \quad \zeta \in C^{n} .
$$

Here we used the duality $\mathscr{B}_{c}\left(\boldsymbol{R}^{n}\right) \leftrightarrow \mathcal{A}\left(\boldsymbol{R}^{n}\right)$. $\hat{\mu}$ becomes an entire function on $\boldsymbol{C}^{n}$. If we set

$$
\begin{aligned}
& G^{n}=\left\{J(\zeta) \in \mathcal{O}\left(\boldsymbol{C}^{n}\right) ; \text { there exists a positive } a \text { such that for any } \varepsilon>0\right. \\
& \left.\qquad \sup _{\zeta \in C^{n}}\left|e^{-\varepsilon|\zeta|-a|\operatorname{Im} \zeta|} J(\zeta)\right|<+\infty\right\}, \\
& G_{0}^{n}=\left\{J(\zeta) \in \mathcal{O}\left(\boldsymbol{C}^{n}\right) ; \sup _{\zeta \in C^{n}}\left|e^{-s|\zeta|} J(\zeta)\right|<+\infty \text { for any } \varepsilon>0\right\},
\end{aligned}
$$

we have bijections:

$$
\begin{aligned}
& \mathscr{I} \mathcal{L}: \mathscr{B}_{c}\left(\boldsymbol{R}^{n}\right) \longrightarrow G^{n}: \mu \longmapsto \hat{\mu}, \\
& \mathscr{I} \mathcal{L}: \mathscr{B}[\{0\}] \longrightarrow G_{0}^{n}: \mu \longmapsto \hat{\mu} .
\end{aligned}
$$


Next we define Fourier transformation on $Q\left(\boldsymbol{D}^{n}\right)$. For $\phi \in Q\left(\mathbb{D}^{n}\right)$, we can define the Fourier transform $\hat{\phi}$ of $\phi$ in $Q\left(D^{n}\right)$ by

$$
\langle\hat{\psi}, \varphi\rangle=\langle\phi, \hat{\varphi}\rangle \quad \text { for any } \varphi \in \mathscr{P}_{*} .
$$

Then we have an isomorphism as (DFS) spaces:

$$
\mathscr{F}: Q\left(\boldsymbol{D}^{n}\right) \stackrel{\sim}{\longrightarrow}\left(\boldsymbol{D}^{n}\right): \phi \longmapsto \hat{\psi} .
$$

Now we give the definition of convolutions. Let $\mu$ be a hyperfunction with compact support. For $f \in \mathscr{B}\left(\boldsymbol{R}^{n}\right)$, we define the convolution $\mu * f$ of $\mu$ and $f$ by

$$
(\mu * f)(x)=\int \mu(x-y) f(y) d y \in \mathscr{B}\left(\boldsymbol{R}^{n}\right) .
$$

The convolutions of $\mu$ and Fourier hyperfunctions are defined by using the duality $Q\left(\boldsymbol{D}^{n}\right) \leftrightarrow \mathscr{Q}_{*}$. First we remark that

and that the map

$$
\mu * \mathscr{P}_{*} \subset \mathscr{P}_{*},
$$

$$
\mu *: \mathscr{Q}_{*} \longrightarrow \mathscr{P}_{*}
$$

is continuous. Taking into account of this, we give

Definition 1.2 (Convolution of $\mu$ and Fourier hyperfunctions). For $\mu \in$ $\mathscr{B}_{c}\left(\boldsymbol{R}^{n}\right)$, we set

$$
\nu(x)=\mu(-x) .
$$

Then for $\psi \in Q\left(\boldsymbol{D}^{n}\right)$, we define the convolution of $\mu$ and $\phi$ in $Q\left(\boldsymbol{D}^{n}\right)$ by

$$
\langle\mu * \psi, \varphi\rangle=\langle\psi, \nu * \varphi\rangle \quad \text { for any } \varphi \in \mathscr{Q}_{*} .
$$

We give a formula on the relation between Fourier transformation and convolutions.

Proposition 1.3. For $\mu \in \mathscr{B}_{c}\left(\boldsymbol{R}^{n}\right)$ and $\phi \in Q\left(\mathbb{D}^{n}\right)$, we have

$$
\widehat{\mu * \phi}=\hat{\mu} \cdot \hat{\psi} \text {. }
$$

\subsection{Ultradistributions}

First we recall the definitions of the classes $\mathcal{E}^{(s)}$ and $\mathcal{E}^{(s)}$ of ultradifferentiable functions for $s>1$. Let $\Omega$ be an open subset of $\boldsymbol{R}^{n}$. For $\varphi(x) \in \mathcal{C}^{\infty}(\Omega)$, $h>0$ and a compact subset $K$ of $\Omega$, we set

$$
\|\varphi\|_{K, h, s}=\sup _{x \in K, a \geqq 0} \frac{\left|D^{a} \varphi(x)\right|}{h^{|a|} \cdot|\alpha !|^{s}} .
$$

Then the space of ultradifferentiable functions of class $(s)$ and that of class $\{s\}$ are given by 


$$
\begin{aligned}
\mathcal{E}^{(s)}(\Omega)= & \left\{\varphi \in \mathcal{C}^{\infty}(\Omega) ;\|\varphi\|_{K, h, s}<+\infty,\right. \\
& \text { for any compact subset } K \text { of } \Omega \text { and any positive } h\}, \\
\mathcal{E}^{(s)}(\Omega)= & \left\{\varphi \in \mathcal{C}^{\infty}(\Omega) ; \text { for any compact subset } K \text { of } \Omega,\right. \\
& \text { there exists a positive } \left.h \text { such that }\|\varphi\|_{K, h, s}<+\infty\right\} .
\end{aligned}
$$

Moreover we define subspaces of the above spaces consisting of functions with compact support :

$$
\begin{aligned}
& \mathscr{D}^{(s)}(\Omega)=\left\{\varphi \in \mathcal{E}^{(s)}(\Omega) ; \quad \operatorname{supp} \varphi \text { is compact }\right\}, \\
& \mathscr{D}^{(s)}(\Omega)=\left\{\varphi \in \mathcal{E}^{(s)}(\Omega) ; \quad \operatorname{supp} \varphi \text { is compact }\right\} .
\end{aligned}
$$

If we set Banach spaces $Y_{K, h, s}$ by

$$
Y_{K, h, s}=\left\{\varphi \in \mathcal{C}^{\infty}\left(\boldsymbol{R}^{n}\right) ; \operatorname{supp} \varphi \subset K,\|\varphi\|_{K, h, s}<+\infty\right\},
$$

then we can express $\mathscr{D}^{(s)}(\Omega)$ and $\mathscr{D}^{(s)}(\Omega)$ as

$$
\begin{aligned}
& \mathscr{D}^{(s)}(\Omega)=\varliminf_{K \Subset \Omega} \lim _{h>0} Y_{K, h, s} . \\
& \mathscr{D}^{(s)}(\Omega)=\lim _{K \in \Omega} \underset{h>0}{\lim _{h, h, s}} Y_{K,}
\end{aligned}
$$

and introduce the topologies of them.

Now we give the definitions of the spaces of ultradistributions. We define the spaces $\mathscr{D}^{(s)^{\prime}}(\Omega)$ and $\mathscr{D}^{(s)^{\prime}}(\Omega)$ as the dual spaces of $\mathscr{D}^{(s)}(\Omega)$ and $\mathscr{D}^{(s)}(\Omega)$ respectively. Elements of $\mathscr{D}^{(s)^{\prime}}(\Omega)$, (resp. $\mathscr{D}^{(s)^{\prime}}(\Omega)$ ) are called ultradistributions of class $(s)$ (resp. $\{s\}$ ).

We give several remarks.

i) $\mathscr{D}^{(s)}(\Omega)$ and $\mathscr{D}^{(s)}(\Omega)$ are not $\{0\}$. Precisely we have the inclusions with dense images:

$$
\mathscr{D}^{\left(s_{0}\right)}(\Omega) \subsetneq \mathscr{D}^{\left(s_{0}\right)}(\Omega) \subsetneq \mathscr{D}^{\left(s_{1}\right)}(\Omega)
$$

for $1<s_{0}<s_{1}$.

ii) The presheaf $\mathscr{D}^{(s)^{\prime}}\left(\right.$ resp. $\left.\mathscr{D}^{(s)^{\prime}}\right): \Omega \mapsto \mathscr{D}^{(s)^{\prime}}(\Omega)$ (resp. $\left.\mathscr{D}^{(s)^{\prime}}(\Omega)\right)$ becomes a sheaf. Moreover we have the injections:

$$
\mathscr{D}^{\left(s_{0}\right)^{\prime}} \longleftrightarrow \mathscr{D}^{\left(s_{0}\right\}^{\prime}} \longleftrightarrow \mathscr{D}^{\left(s_{1}\right)^{\prime}}
$$

for $1<s_{0}<s_{1}$. Thus we can define the supports of ultradistributions.

\subsection{Kawai's abstract sufficient condition $(\mathbf{S})$}

In this section we introduce a sufficient condition (S) for the surjectivity on the space of hyperfunctions. We will utilize the expression of hyperfunctions by boundary values of holomorphic functions. As far as the surjectivity is concerned, we can prove

Claim. Let $\mu$ be a hyperfunction with compact support. Let $\Gamma_{\sigma}\left(\sigma \in\{-1,1\}^{n}\right)$ 
be an open subset in $\boldsymbol{R}^{n}$ of the form

$$
\Gamma_{\sigma}=\left\{y \in \boldsymbol{R}^{n} ; \sigma_{j} \cdot y_{j}>0,(j=1, \cdots, n)\right\} .
$$

Assume that for any $\Gamma_{\sigma}$, the operator $\mu *$ is surjective on $\mathcal{O}\left(\boldsymbol{R}^{n} \times \sqrt{-1} \Gamma_{\sigma}\right)$. Then $\mu *$ is surjective on the space $\mathcal{B}\left(\boldsymbol{R}^{n}\right)$.

For instance let us consider the case $\Gamma=\left\{y \in \boldsymbol{R}^{n} ; y_{0}>0, \quad(j=1, \cdots, n)\right\}$. Then we can regard the space $\mathcal{O}\left(\boldsymbol{R}^{n} \times \Gamma\right)$ as an (FS) space in a usual way, and it is possible to make a use of the theory of locally convex spaces. In this direction, Kawai [Ka-2] proved the following theorem.

Theorem 1.4. Let $\mu$ be a compact supported hyperfunction whose FourierLaplace transform $\hat{\mu}$ satisfies the condition:

$$
\left\{\begin{array}{l}
\forall \varepsilon>0, \exists N_{\varepsilon}>0, \forall \xi \in \boldsymbol{R}^{n} \quad \text { with }|\xi|>N_{\varepsilon}, \exists \eta \in C^{n}, \\
\text { satisfying } \\
\text { i) }|\xi-\eta|<\varepsilon|\xi|, \\
\text { ii) }|\hat{\mu}(\eta)| \geqq e^{-\varepsilon|\xi|} .
\end{array}\right.
$$

Then the convolution operator

is surjective.

$$
\mu *: \mathscr{B}\left(\boldsymbol{R}^{n}\right) \longrightarrow \mathscr{B}\left(\boldsymbol{R}^{n}\right)
$$

\section{$\S 2$. Surjectivity of Convolution Operators with Ultradistribution Kernel on the Space of Hyperfunctions}

\subsection{Statement of the theorem on the space of hyperfunctions}

In this chapter we will show the surjectivity of convolution operators with ultradistribution kernel on the space $\mathscr{B}\left(\boldsymbol{R}^{n}\right)$. Utilizing Theorem 1.4 of abstract nature, we give

Theorem 2.1. Let $\mu$ be a non-zero ultradistribution with compact support. Then

$$
\mu *: \mathscr{B}\left(\boldsymbol{R}^{n}\right) \longrightarrow \mathscr{B}\left(\boldsymbol{R}^{n}\right)
$$

is surjective.

We will prove the above theorem in the special case $n=1$ in $\S 2.2$ and the general case in $\S 2.3$.

Precisely, we will show that $\hat{\mu}$ satisfies the condition: 


$$
\left\{\begin{array}{l}
\forall \varepsilon>0, \exists N_{\varepsilon}>0, \forall \xi \in \boldsymbol{R}^{n} \quad \text { with }|\xi|>N_{s}, \exists \eta \in \boldsymbol{R}^{n}, \\
\text { satisfying } \\
\text { i) }|\xi-\eta|<\varepsilon|\xi|, \\
\text { ii) }|\hat{\mu}(\eta)| \geqq e^{-\varepsilon|\xi|},
\end{array}\right.
$$

which is a slightly stronger condition than (S). We can regard (S0) as a condition for functions on $\boldsymbol{R}^{n}$. Moreover if $J$ is entire and if $\left.J\right|_{R^{n}}$ satisfies (S0), then $J$ satisfies (S).

\subsection{One dimensional case}

First we study the case $n=1$. This will make it easier to study the general case. We will show the following theorem in this section.

Theorem 2.2. Let $\mu$ be a non-zero ultradistribution with compact support of one variable. Then $\hat{\mu}$ satisfies the condition (S0).

Proof. Let $\mu$ be as above. Then there exist constants $s$ and $a$ such that

$$
\begin{aligned}
& \text { i) } s>1, \quad a>0, \\
& \text { ii) } \mu \in \mathscr{D}^{(s)^{\prime}}(\boldsymbol{R}), \\
& \text { iii) } \operatorname{supp} \mu \subset[-a, a] .
\end{aligned}
$$

Here we quote two theorems from Komatsu [Ko-3] and Boas [Bo].

Theorem 2.3 (Paley-Wiener type). Let $\mu$ be an element of $\mathscr{D}^{(s)^{\prime}}(\boldsymbol{R})$ and assume supp $\mu \subset[-a, a]$. Then there exist constants $L>0$ and $C>0$ satisfying the estimate

$$
|\hat{\mu}(\zeta)| \leqq C \exp \left\{(L|\zeta|)^{1 / s}+a|\operatorname{Im} \zeta|\right\} .
$$

Remark that the inverse also holds. But we will not use it.

Theorem 2.4 (Theorem 6.3.6 of Boas [Bo]). Let $f$ be an entire function satisfying the estimates

$$
\begin{gathered}
\liminf _{r \rightarrow \infty} r^{-1} \log \left(\max _{|z|=r}|f(z)|\right)<\infty, \\
\int_{-\infty}^{\infty} \frac{\max (0, \log |f(x)|)}{1+x^{2}} d x<\infty .
\end{gathered}
$$

Then $f$ satisfies

$$
\int_{-\infty}^{\infty} \frac{|\log | f(x)||}{1+x^{2}} d x<\infty
$$

Note that the consequence of Theorem 2.4 is equivalent to the condition: 


$$
\int_{-\infty}^{\infty} \frac{\log |f(x)|}{1+x^{2}} d x>-\infty
$$

Lemma 2.5. Let $f$ be a function on $\boldsymbol{R}$ which satisfies

$$
\int_{-\infty}^{\infty} \frac{\log |f(\xi)|}{1+\xi^{2}} d \xi>-\infty
$$

then $f$ satisfies the condition ( $\mathrm{S} 0$ ).

Proof of Lemma 2.5. Assume that $f$ does not satisfy (S0). Then there exist sufficiently small $\varepsilon>0$ and $\xi_{n} \in \boldsymbol{R}(n=1,2, \cdots)$ such that

If we set

$$
\begin{gathered}
\left|\xi_{n}\right| \geqq \frac{1+\varepsilon}{1-\varepsilon}\left|\xi_{n-1}\right|, \\
\left|\eta-\xi_{n}\right|<\varepsilon\left|\xi_{n}\right| \text { implies }|f(\eta)|<e^{-\varepsilon_{1} \xi_{n} \mid} .
\end{gathered}
$$

then we have

$$
\left.I_{n}=\right] \xi_{n}-\varepsilon\left|\xi_{n}\right|, \quad \xi_{n}+\varepsilon\left|\xi_{n}\right|[\quad(n=1,2, \cdots),
$$

$$
|f|<e^{-\varepsilon\left|\xi_{n}\right|} \quad \text { on } I_{n} \text { for any } n,
$$

Since $n \neq m$ implies $I_{n} \cap I_{m}=\emptyset$, we get

$$
\begin{aligned}
\int_{-\infty}^{\infty} \min \left(0, \frac{\log |f(\xi)|}{1+\xi^{2}}\right) d \xi & \leqq \sum_{n=1}^{\infty} \int_{I_{n}} \frac{-\varepsilon\left|\xi_{n}\right|}{1+\xi^{2}} d \xi \\
& \leqq \sum_{n=1}^{\infty} \frac{-2 \varepsilon^{2}\left|\xi_{n}\right|^{2}}{1+(1+\varepsilon)^{2}\left|\xi_{n}\right|^{2}}
\end{aligned}
$$

Remark that

$$
\frac{-2 \varepsilon^{2}\left|\xi_{n}\right|^{2}}{1+(1+\varepsilon)^{2}\left|\xi_{n}\right|^{2}}=\frac{-2 \varepsilon^{2}}{\frac{1}{\left|\xi_{n}\right|^{2}}+(1+\varepsilon)^{2}} \stackrel{n \rightarrow \infty}{\longrightarrow} \frac{-2 \varepsilon^{2}}{(1+\varepsilon)^{2}}<0 .
$$

Thus the right hand side of the above inequalities diverges to $-\infty$, and it contradicts the assumption of this lemma. (q. e.d. for Lemma 2.5.)

Under the above preparation, we can show Theorem 2.2. By Theorem 2.3, $\hat{\mu}$ has the estimates

$$
\begin{aligned}
& \log |\hat{\mu}(\zeta)| \leqq C_{1}+C_{2}|\zeta|, \quad(\boldsymbol{\zeta} \in \boldsymbol{C}), \\
& \log |\hat{\mu}(\xi)| \leqq C_{1}+C_{2}|\xi|^{1 / s}, \quad(\xi \in \boldsymbol{R}),
\end{aligned}
$$

for some constants $C_{1}$ and $C_{2}$. Then $\hat{\mu}$ satisfies the assumption of Theorem 2.4. Now we get Theorem 2.2 from Theorem 2.4 and Lemma 2.5. (q.e.d. for Theorem 2.2.)

Remark 2.6. We can derive the following consequence by a similar argument. If $\mu$ is in a non quasianalytic class, $\hat{\mu}$ satisfies the condition (S0). See 
L. Ehrenpreis ([E-2]§V.6). This fact also holds even in case $n \geqq 2$.

\subsection{General case}

Let $\mu$ be a non-zero ultradistribution with compact support of $n$ variables. We will show in this section that $\hat{\mu}$ satisfies the condition (S0) also in the general case $n \geqq 2$.

Lemma 2.7. Let $Z$ be a subset of $S^{n-1}=\left\{\xi \in \boldsymbol{R}^{n} ;|\boldsymbol{\xi}|=1\right\}$ satisfying that $S^{n-1} \backslash Z$ is dense in $S^{n-1}$. For a function $f$ defined on $\boldsymbol{R}^{n}$ and $\xi \in S^{n-1}$, we define a function $\varphi_{\xi}$ of one variable $\tau \in \boldsymbol{R}$ by

$$
\varphi_{\xi}(\tau)=f(\tau \xi) \quad(\tau \in \boldsymbol{R}) .
$$

Assume that for any $\xi \in S^{n-1} \backslash Z, \varphi_{\xi}$ satisfies the codition (S0) as a function of one variable. Then $f$ satisfies the condition ( $\mathrm{S} 0)$ as a function of $n$ variables.

Proof. Take any $\varepsilon>0$. We fix an element $\xi$ of $S^{n-1} \backslash Z$. Then it follows from the assumption of the lemma that

$$
\left\{\begin{aligned}
& \exists N_{\xi, \varepsilon}, \forall \tau \in \boldsymbol{R} \text { with }|\tau|>N_{\xi, \varepsilon}, \exists \rho \in \boldsymbol{R}, \\
& \text { satisfying } \\
& \text { i) } \quad|\tau-\rho|<\frac{\varepsilon}{2}|\tau|, \\
& \text { ii) }\left|\varphi_{\xi}(\rho)\right| \geqq e^{-\varepsilon / 2|\tau|} .
\end{aligned}\right.
$$

We set

$$
B_{\boldsymbol{R}^{n}}(\xi ; r)=\left\{\eta \in \boldsymbol{R}^{n} ;|\eta-\xi|<r\right\},
$$

and consider a family of open balls

$$
\left\{B_{R^{n}}\left(\xi ; \frac{\varepsilon}{2}\right)\right\}_{\xi \in S^{n-1} \backslash Z}
$$

Since $S^{n-1} \backslash Z$ is dense in $S^{n-1}$, we have

$$
S^{n-1} \subset \underset{\xi \in S^{n-1} \backslash Z}{\bigcup} B_{R n}\left(\xi ; \frac{\varepsilon}{2}\right) .
$$

From the compactness of $S^{n-1}$, we can take $\xi_{1}, \xi_{2}, \cdots, \xi_{m} \in S^{n-1} \backslash Z$ such that

$$
S^{n-1} \subset \bigcup_{k=1}^{m} B_{R n}\left(\xi_{k} ; \frac{\varepsilon}{2}\right) \text {. }
$$

Now we set

$$
N_{\varepsilon}=\max _{k=1, \cdots, m} N_{\xi_{k}, \varepsilon},
$$

and take any $\xi \in \boldsymbol{R}^{n}$ with $|\xi|>N_{\varepsilon}$. Then there exists $k \in\{1, \cdots, m\}$ such that

$$
\frac{\xi}{|\xi|} \in B_{R n}\left(\xi_{k} ; \frac{\varepsilon}{2}\right) \text {. }
$$


Note that

$$
\begin{aligned}
& |\xi-| \xi\left|\cdot \xi_{k}\right|=|\xi| \cdot\left|\frac{\xi}{|\xi|}-\xi_{k}\right|<\frac{\varepsilon}{2}|\xi|, \\
& || \xi\left|\cdot \xi_{k}\right|=|\xi|>N_{\varepsilon} \geqq N_{\xi_{k}, \varepsilon} .
\end{aligned}
$$

Hence we can find $\rho \in \boldsymbol{R}$ such that

$$
|\rho-| \xi||<\frac{\varepsilon}{2}|\xi|, \quad\left|\varphi_{\xi_{k}}(\rho)\right| \geqq e^{-\varepsilon / 2|\xi|} .
$$

This implies that

$$
\begin{aligned}
& \left|\xi-\rho \cdot \xi_{k}\right| \leqq|\xi-| \xi\left|\cdot \xi_{k}\right|+|| \xi\left|\cdot \xi_{k}-\rho \cdot \xi_{k}\right|<\varepsilon|\xi|, \\
& \left|f\left(\rho \cdot \xi_{k}\right)\right|=\left|\varphi_{\xi_{k}}(\rho)\right| \geqq e^{-\varepsilon / 2|\xi|} \geqq e^{-\varepsilon|\xi|} .
\end{aligned}
$$

(q.e.d. for Lemma 2.7.)

Now we can give the proof of Theorem 2.2.

Proof of Theorem 2.2. Let $\mu$ be a non-zero ultradistribution of $n$ variable. If we set

we have

$$
\begin{aligned}
f(\xi) & =\left\langle\mu, e^{-i x \xi}\right\rangle_{x}, \\
\varphi_{\xi}(\tau) & =f(\tau \cdot \xi),
\end{aligned}
$$

$$
\begin{aligned}
\varphi_{\xi}(\tau) & =\left\langle\mu, e^{-i x \xi \cdot \tau}\right\rangle_{x} \\
& =\int_{R^{n}} \mu(x) e^{-i(x \xi) \cdot \tau} d x \\
& =\int_{R} \nu_{\xi}(t) e^{-i t \tau} d t .
\end{aligned}
$$

Here

$$
\nu_{\xi}(t)=\int_{x \cdot \xi=t} \mu(x) d \tilde{x},
$$

and $d \tilde{x}$ is the standard $(n-1)$-form on $\boldsymbol{R}^{n}$ which satisfies $d \tilde{x} \wedge d t=d x$. Then $\varphi_{\xi}$ is the Fourier transform of the compactly supported ultradistribution $\nu_{\xi}$. As a consequence of Theorem 2.2 of one dimensional case, we get

$$
\varphi_{\xi} \equiv 0 \text {, or } \varphi_{\xi} \text { satisfies (S0). }
$$

Since $f$ is analytic, we get

$$
Z=\left\{\xi \in S^{n-1} ; \varphi_{\xi} \equiv 0\right\}
$$

is nowhere dense in $S^{n-1}$. Then the pair $(f, Z)$ satisfies the assumption of Lemma 2.7. This implies the desired result. (q. e. d. for Theorem 2.2.) 
Remark 2.8. Now let $\mu$ be a non-zero distribution with compact support. As a consequence of Theorem 2.2, we find the operator $\mu *$ surjective on $\mathscr{B}\left(\boldsymbol{R}^{n}\right)$.

Next we begin to study the case that the support of $\mu$ is one point. The surjectivity of $\mu *$ can be also reduced to the condition (S). First by translation, we may assume that supp $\mu=\{0\}$. Moreover by an argument similar to Lemma 2.7 for the condition (S), it is sufficient to show

Claim. Let $\mu$ be a hyperfunction of one variable whose support is the origin. Then the Fourier-Laplace transform $\hat{\mu}$ of $\mu$ satisfies the condition (S).

If we remark that $\hat{\mu}$ is contained in $G_{0}^{1}$, Claim is an easy corollary to the following theorem in Levin ([L] p. 21, Th. 11).

Theorem 2.9. Let $f$ be a holomorphic function in the circle $\{z \in C ;|z| \leqq 2 e R\}$ $(R>0)$ with $f(0)=1$, and let $\eta$ be an arbitrary positive number not exceeding 3e/2. Then there exists a family $\left\{B_{C}\left(z_{k} ; r_{k}\right)\right\}_{k=1}^{m}$ of circles in $C$ such that

$$
\sum_{k} r_{k} \leqq 4 \eta R
$$

and that the estimate

$$
\log |f(z)|>-\left(2+\log \frac{3 e}{2 \eta}\right) \log \left\{\max _{\mid w_{i=2 e}}|f(w)|\right\}
$$

holds in the domain

$$
\{z \in C ;|z| \leqq R\} \backslash \bigcup_{k=1}^{m} B_{C}\left(z_{k} ; r_{k}\right)
$$

\section{$\S 3$. Surjectivity of Convolution Operators on the Space of Fourier Hyperfunctions}

\subsection{Statement of the theorems on the space of Fourier hyperfunctions}

In this chapter we consider convolution operators on the space $Q\left(\boldsymbol{D}^{n}\right)$. First we give necessary conditions for the surjectivity, one for the case $n=1$, the other for the general case.

Theorem 3.1. Let $\mu$ be a hyperfunction with compact support of one variable. Assume that the convolution operator

$$
\mu *: Q\left(D^{1}\right) \longrightarrow Q\left(D^{1}\right)
$$

is surjective. Then there exists a positive number $\delta$ for which the set

$$
\{\zeta \in \mathbb{C} ; 0<|\operatorname{Im} \zeta|<\delta, \hat{\mu}(\zeta)=0\}
$$

is empty.

Theorem 3.2. Let $\mu$ be a hyperfunction with compact support of $n$ variables. 
Assume that the convolution operator

$$
\mu *: Q\left(\boldsymbol{D}^{n}\right) \longrightarrow Q\left(\boldsymbol{D}^{n}\right)
$$

is surjective. Then the Fourier-Laplace transform $\hat{\mu}$ of $\mu$ satisfies the condition:

$$
\left\{\begin{aligned}
\forall \varepsilon>0, \exists N_{\varepsilon}>0, \forall \xi \in R^{n} \text { with }|\xi|>N_{\varepsilon}, \exists \eta \in C^{n}, \\
\text { satisfying } \\
\text { i) }|\xi-\eta|<\varepsilon, \\
\text { ii) }|\hat{\mu}(\eta)| \geqq e^{-\varepsilon|\xi|} .
\end{aligned}\right.
$$

Next let us consider an inverse of Theorem 3.1 and Theorem 3.2.

Theorem 3.3. Let $\mu$ be a hyperfunction with compact support whose FourierLaplace transform $\hat{\mu}$ satisfies the condition (S') and the condition:

$$
\left\{\begin{array}{l}
\exists \delta>0, \forall \delta^{\prime} \text { with } 0<\delta^{\prime}<\delta, \\
\text { all the irreducible components of the analytic variety } \\
\quad\left\{\zeta \in \boldsymbol{C}^{n} ;|\operatorname{Im} \zeta|<\delta^{\prime}, \hat{\mu}(\zeta)=0\right\} \\
\text { intersect with } \boldsymbol{R}^{n} .
\end{array}\right.
$$

Then the convolution operator

is surjective.

$$
\mu *: Q\left(\boldsymbol{D}^{n}\right) \longrightarrow Q\left(\boldsymbol{D}^{n}\right)
$$

Note that in case $n=1$, the condition ( $\left.Z^{\prime}\right)$ is equivalent to the conclusion in Theorem 3.1.

Corollary. In case $n=1$, the surjectivity of $\mu *$ is equivalent to ( $\left.\mathrm{S}^{\prime}\right)$ and ( $\left.\mathrm{Z}^{\prime}\right)$.

The following theorem asserts the independence of ( $\left.\mathrm{S}^{\prime}\right)$ and $\left(Z^{\prime}\right)$.

\section{Theorem 3.4.}

i) There exists a hyperfunction $\mu$ of one variable supported in the origin such that $\hat{\mu}$ satisfies ( $\left.\mathrm{S}^{\prime}\right)$ and does not satisfy (Z').

ii) There exists a hyperfunction $\mu$ supported in the origin such that $\hat{\mu}$ satisfies (Z') and does not satisfy ('').

Corollary. There exists a non-zero hyperfunction $\mu$ supported in the origin such that the convolution operator

is not surjective.

$$
\mu *: Q\left(\boldsymbol{D}^{n}\right) \longrightarrow Q\left(\boldsymbol{D}^{n}\right)
$$

Note that a convolution operator with a kernel supported in the origin be- 
comes a local operator, i. e., a differential operator of infinite order with constant coefficients.

We give some remarks.

(i) The condition ( $\mathrm{S}^{\prime}$ ) is due to $\mathrm{T}$. Kawai [Ka-2]. He introduced this condition as a sufficient condition for surjectivity on $Q\left(\boldsymbol{D}^{n}\right)$. But his proof seems to have forgotten to estimate the domain of holomorphy of functions divided by $\hat{\mu}$. In general, we need the condition ( $S^{\prime}$ ) for surjectivity. Moreover in case $n=1$, we deduce the condition ( $\mathrm{S}^{\prime}$ ) and ( $\left.Z^{\prime}\right)$ as a necessary and sufficient condition for surjectivity of $\mu *$. But it seems difficult to the author to get a necessary and sufficient condition in case $n \geqq 2$, since analytic varieties of codimension 1 never have isolated points.

(ii) Theorem 3.3 also holds under weaker conditions (S') and (Z') by the same proof. Here

$$
\left\{\begin{array}{l}
\exists \delta>0, \forall \delta^{\prime} \quad \text { with } 0<\delta^{\prime}<\delta, \\
\text { all the irreducible components of the analytic variety } \\
\qquad\left\{\zeta \in C^{n} ;|\operatorname{Im} \zeta|<\delta^{\prime}, \hat{\mu}(\zeta)=0\right\} \\
\text { intersect with } \boldsymbol{R}^{n} \text { with finite exceptions. }
\end{array}\right.
$$

This fact is suggested by Prof. A. Kaneko.

\subsection{Proof of the theorems}

In considering convolution operators on the space $Q\left(\boldsymbol{D}^{n}\right)$, we can develop Fourier analysis exactly; that is, we can transform a convolution operator

$$
\mu *: Q\left(\boldsymbol{D}^{n}\right) \longrightarrow Q\left(\boldsymbol{D}^{n}\right)
$$

into a multiplication operator

$$
\hat{\mu} \cdot: Q\left(\boldsymbol{D}^{n}\right) \longrightarrow Q\left(\boldsymbol{D}^{n}\right)
$$

by Fourier transformation. Thus the surjectivity of $\mu *$ is equivalent to that of $\hat{\mu} \cdot$, and we rewrite the theorems in the last section in terms of multiplications by holomorphic functions.

Theorem 3.1'. Let $J(\zeta)$ be an element of $G^{1}$. Assume that the multiplication operator

$$
J \cdot: Q\left(\boldsymbol{D}^{1}\right) \longrightarrow Q\left(\boldsymbol{D}^{1}\right)
$$

is surjective. Then there exists a positive number $\delta$ for which the set

is empty.

$$
\{\zeta \in C ; 0<|\operatorname{Im} \zeta|<\delta, J(\zeta)=0\}
$$

Theorem 3.2'. Let $J(\zeta)$ be an element of $G^{n}$. Assume that the multiplication 
operator

$$
J \cdot: Q\left(\mathbb{D}^{n}\right) \longrightarrow Q\left(\mathbb{D}^{n}\right)
$$

is surjective. Then $J$ satisfies the condition:

$$
\left\{\begin{array}{l}
\forall \varepsilon>0, \exists N_{\varepsilon}>0, \forall \xi \in \mathbb{R}^{n} \quad \text { with }|\xi|>N_{\varepsilon}, \exists \eta \in C^{n}, \\
\text { satisfying } \\
\text { i) }|\xi-\eta|<\varepsilon, \\
\text { ii) }|J(\eta)| \geqq e^{-\varepsilon|\xi|} .
\end{array}\right.
$$

Theorem 3.3'. Let $J(\zeta)$ be an element of $G^{n}$ and satisfy the condition (S') and the condition:

$$
\left\{\begin{array}{l}
\exists \delta>0, \forall \delta^{\prime} \text { with } 0<\delta^{\prime}<\delta, \\
\text { all the irreducible components of the analytic variety } \\
\quad\left\{\zeta \in \mathbb{C}^{n} ;|\operatorname{Im} \zeta|<\delta^{\prime}, J(\zeta)=0\right\} \\
\text { intersect with } \mathbb{R}^{n} \text {. }
\end{array}\right.
$$

Then the multiplication operator

$$
J \cdot: Q\left(\mathbb{D}^{n}\right) \longrightarrow Q\left(\mathbb{D}^{n}\right)
$$

is surjective.

\section{Theorem 3.4'。}

i) There exists $J \in G_{0}^{1}$ which satisfies ( $\left.\mathrm{S}^{\prime}\right)$ and does not satisfy ( $\left.Z^{\prime}\right)$.

ii) There exists $J \in G_{0}^{n}$ which satisfies ( $\left.Z^{\prime}\right)$ and does not satisfy ('').

We prepare several lemmas. First we cite two lemmas from the theory of (FS) and (DFS) spaces. See Komatsu [Ko-1] for their proof.

Lemma 3.5. Let $X$ be a (DFS) space and $Y$ be the strong dual space of $X$, which is an (FS) space. Let $T: X \rightarrow X$ be a linear continuous operator and $T^{\prime}$ : $Y \rightarrow Y$ be the dual operator of $T$. Then the following three conditions are equivalent.

i) $T^{\prime}$ is surjective.

ii) $T$ is injective and the range $R(T)$ of $T$ is closed subspace of $X$.

iii) $T: X \rightarrow R(T)$ is an isomorphism between two locally convex spaces.

Lemma 3.6. Let $X_{j}(j=1,2, \cdots)$ be Banach spaces, $i_{j}: X_{j} \rightarrow X_{j+1}(j=1,2, \cdots)$ injective linear compact maps, and $X$ the inductive limit of the system $\left\{X_{j}, i_{j}\right\}_{j \in N}$. Thus we can regard $X_{j}$ as subspaces of $X$. Then the following two conditions for a sequence $\left\{x_{k}\right\}_{k=1}^{\infty}$ in $X$ are equivalent.

i) $x_{k} \rightarrow 0$ in the strong topology (resp. weak topology) of $X$. 
ii) For some $j,\left\{x_{k}\right\}_{k}$ is contained in $X_{j}$ and $x_{k} \rightarrow 0$ in the strong topology (resp. weak topology) of $X_{j}$.

In our situation, the (DFS) space $\mathscr{L}_{*}$ is expressed as the inductive limit of Banach spaces:

$$
\mathscr{Q}_{*}=\varliminf_{j \rightarrow \infty} X_{j} .
$$

Here $X_{j}$ 's are Banach spaces endowed with norms $\|\cdot\|_{\mathcal{J}}(j=1,2, \cdots)$ :

$$
\begin{aligned}
X_{j}= & \left\{\varphi \in \mathcal{O}\left(\boldsymbol{R}^{n} \times \sqrt{-1} B_{R n}\left(0 ; \frac{1}{j}\right)\right) ;\|\varphi\|_{j}<+\infty,\right. \\
& \left.\varphi \text { extends continuously to the closure of } \boldsymbol{R}^{n} \times \sqrt{-1} B_{\boldsymbol{R} n}\left(0 ; \frac{1}{j}\right) \text { in } \boldsymbol{C}^{n}\right\}, \\
\|\varphi\|_{j}= & \sup _{\mid \operatorname{lm} \zeta<1 / \jmath}\left|\exp \left(\frac{1}{j}|\operatorname{Re} \zeta|\right) \varphi(\zeta)\right| .
\end{aligned}
$$

Next we quote two lemmas from the theory of holomorphic functions.

Lemma 3.7. Let $f(z)$ and $g^{\prime}(z)$ be holomorphic functions defined in a neighborhood of $\{|z| \leqq R\}$ with the estimates

$$
|f(z)|<A, \quad|g(z)|<B, \quad(|z| \leqq R),
$$

for some positive constants $A$ and $B$. Assume that $f(z) / g(z)$ is holomorphic on the same domain. Then we get the estimate

$$
|f(z) / g(z)| \leqq A B^{2}|g(0)|^{-3}, \quad\left(|z| \leqq \frac{R}{2}\right) .
$$

See Kawai [Ka-1].

Lemma 3.8. Let $\left\{z_{k}\right\}_{k=1}^{\infty}$ be a sequence in $C$ with the estimate

$$
\sum_{k=1}^{\infty} \frac{1}{\left|z_{k}\right|}<+\infty
$$

We set

$$
f(z)=\prod_{k=1}^{\infty}\left(1-\frac{z}{z_{k}}\right) .
$$

Then the infinite product converges on any compact subset of $\boldsymbol{C} \backslash\left\{z_{k}\right\}_{k}$, and $f$ becomes an entire function with the properties:

i) $f$ is contained in $G_{0}^{1}$,

ii) the zero set of $f$ coincides with $\left\{z_{k}\right\}_{k}$.

See Levin [L].

From now on, we will give the proof of the theorems.

Proof of Theorem 3.1'. We will prove this theorem by contradiction. 
Assume that there exists a sequence $\left\{\zeta_{k}\right\}_{k=1}^{\infty}$ in $C$ satisfying

$$
\begin{array}{ll}
\text { 1) } \quad 0<\left|\operatorname{Im} \zeta_{k}\right|<\frac{1}{k} & (k=1,2, \cdots), \\
\text { 2) } J\left(\zeta_{k}\right)=0 & (k=1,2, \cdots) .
\end{array}
$$

Then on account of Lemma 3.5, it is sufficient to construct a sequence $\left\{\varphi_{k}\right\}$ in $\mathscr{P}_{*}$ such that

i) the sequence $\left\{\varphi_{k}\right\}$ does not converge in $\mathscr{L}_{*}$,

ii) the sequence $\left\{J \cdot \varphi_{k}\right\}$ converges to 0 in $\mathscr{Q}_{*}$.

For this purpose we put

$$
\varphi_{k}(\zeta)=\frac{e^{-\zeta^{2}}}{k\left(\zeta-\zeta_{k}\right)} .
$$

Then it is easy to see that $\varphi_{k}$ 's are contained in $\mathscr{P}_{*}$. Moreover taking into account of the domains of holomorphy of $\varphi_{k}$ 's, we can deduce from Lemma 3.6 that the sequence $\left\{\varphi_{k}\right\}$ does not converge in $\mathscr{Q}_{*}$.

On the other hand, the sequence $\left\{J \cdot \varphi_{k}\right\}$ converges to 0 in $\mathscr{P}_{*}$. In fact, $J(\zeta) / \zeta-\zeta_{k}$ is entire, and there exists a positive constant $C$ for which $\left\{J(\zeta) / \zeta-\zeta_{k}\right\}_{k}$ satisfy the estimate

$$
\left|\frac{J(\zeta)}{\zeta-\zeta_{k}}\right| \leqq C e^{|\operatorname{Re} \zeta|} \quad(k=1,2, \cdots)
$$

on $\{\zeta \in C ;|\operatorname{Im} \zeta|<1\}$. Thus

$$
J(\zeta) \cdot \varphi_{k}(\zeta)=\frac{e^{-\zeta^{2}}}{k} \cdot \frac{J(\zeta)}{\zeta-\zeta_{k}}
$$

is entire and is estimated on the same domain as

$$
\begin{aligned}
\left|J(\zeta) \cdot \varphi_{k}(\zeta)\right| & \leqq \frac{C}{k} \cdot e^{-\mid \operatorname{Re} \zeta_{1}^{2}+1} \cdot e^{|\operatorname{Re} \zeta|} \\
& =\frac{C e^{-2}}{k} \cdot e^{-(|\operatorname{Re} \zeta|-1)^{2}} \cdot e^{-|\operatorname{Re} \zeta|} \\
& \leqq \frac{C e^{-2}}{k} \cdot e^{-|\operatorname{Re} \zeta|} .
\end{aligned}
$$

From this estimate and Lemma 3.6, we obtain the desired result. (q.e.d. for Theorem 3.1'.)

Proof of Theorem 3.2'. We will prove this theorem by contradiction. Assume that there exists a sequence $\left\{\boldsymbol{\xi}_{k}\right\}_{k=1}^{\infty}$ in $\boldsymbol{R}^{n}$ and positive number $\varepsilon<1$ such that

1) $\left|\xi_{k}\right|>k$,

2) $J(\zeta) \leqq \exp \left\{-2 \varepsilon^{2}\left|\dot{\xi}_{k}\right|\right\} \quad$ on the ball $B_{C n}\left(\xi_{k} ; 2 n \varepsilon\right)(k=1,2, \cdots)$.

Then taking into account of Lemma 3.5, we find it sufficient to construct a 
sequence $\left\{\varphi_{k}\right\}$ in $\mathscr{Q}_{*}$ with the properties

i) the sequence $\left\{\varphi_{k}\right\}$ does not converge in $\mathscr{L}_{*}$,

ii) the sequence $\left\{J \cdot \varphi_{k}\right\}$ converges to 0 in $\mathscr{Q}_{*}$.

For this purpose we put

$$
\varphi_{k}(\zeta)=\frac{1}{k} e^{-\left|\hat{s}_{k}\right|\left(\zeta-\hat{s}_{k}\right) 2} .
$$

Note that $\varphi_{k}$ 's are entire and contained in $\mathscr{P}_{*}$.

First we will show that $\left\{\varphi_{k}\right\}$ does not converge in $\mathscr{P}_{*}$. Assume that $\left\{\varphi_{k}\right\}$ is a convergent series. Then by Lemma 3.6 we can take a constant $\delta>0$ such that

$$
\left\{\sup _{1 \operatorname{Im} \zeta \backslash<\delta}\left|e^{\delta \backslash \operatorname{Re} \zeta \mid} \cdot \varphi_{k}(\zeta)\right|\right\}_{k}
$$

is bounded. But we have $\varphi_{k}\left(\xi_{k}\right)=1 / k$ for any $k$. Hence we get the estimate

$$
\sup _{|\operatorname{Im} \zeta|<\delta}\left|e^{\delta|\operatorname{Re}|} \varphi_{k}(\zeta)\right| \geqq e^{\hat{\jmath}_{1} \operatorname{Re} \check{\zeta}_{k} \mid} \cdot\left|\varphi_{k}\left(\xi_{k}\right)\right| \geqq \frac{1}{k} \cdot e^{\delta k}
$$

The third side of this inequalities diverges.

Next we will show that $\left\{J \cdot \varphi_{k}\right\}$ converges to 0 in $\mathscr{P}_{*}$. For this, we will estimate $\left|J(\zeta) \cdot \varphi_{k}(\zeta)\right|$ in the domain $\left\{\zeta \in C^{n} ;|\operatorname{Im} \zeta|<\varepsilon\right\}$. Since $J$ belongs to $G^{n}$, we have on the above domain

$$
J(\zeta) \leqq C e^{\varepsilon^{2} \mid \operatorname{Re} \zeta}
$$

for some constant $C$. If $\left|\operatorname{Re} \zeta-\xi_{k}\right| \leqq 2 \varepsilon$ and $|\operatorname{Im} \zeta|<\varepsilon$, we have

$$
\begin{aligned}
\left|J(\zeta) \cdot k \varphi_{k}(\zeta)\right| & \leqq e^{-2 \varepsilon^{2}\left|\xi_{k}\right|} \cdot e^{\varepsilon^{2}\left|\xi_{k}\right|} \\
& \leqq e^{-\varepsilon^{2}\left|\xi_{k}\right|} \leqq e^{-\varepsilon^{2}\{|\operatorname{Re} \zeta|-2 \varepsilon\}} \\
& =e^{2 \varepsilon^{3}} \cdot e^{-\varepsilon^{2}|\operatorname{Re} \zeta|}
\end{aligned}
$$

If $\left|\operatorname{Re} \zeta-\xi_{k}\right|>2 \varepsilon$ and $|\operatorname{Im} \zeta|<\varepsilon$, we claim

$$
\left|J(\zeta) \cdot k \varphi_{k}(\zeta)\right| \leqq C e^{-\imath^{2} \mid \operatorname{Re} \zeta} \quad(k=1,2, \cdots) .
$$

In fact we can easily get this from the estimate:

$$
\begin{aligned}
\log \left|k \varphi_{k}(\zeta)\right| & \leqq-\left|\xi_{k}\right|\left|\operatorname{Re} \zeta-\xi_{k}\right|^{2}+\varepsilon^{2}\left|\xi_{k}\right| \\
& \leqq \begin{cases}-2\left|\xi_{k}\right|\left(|\operatorname{Re} \zeta|-\left|\xi_{k}\right|-2 \varepsilon\right)-3 \varepsilon^{2}\left|\xi_{k}\right| & \text { if }|\operatorname{Re} \zeta|>\left|\xi_{k}\right|+2 \varepsilon, \\
-3 \varepsilon^{2}\left|\xi_{k}\right| & \text { if }\left|\operatorname{Re} \zeta-\xi_{k}\right|>2 \varepsilon .\end{cases}
\end{aligned}
$$

After all we get the estimate in the domain $\left\{\zeta \in C^{n} ;|\operatorname{Im} \zeta|<\varepsilon\right\}$

$$
\left|J(\zeta) \cdot k \varphi_{k}(\zeta)\right| \leqq C^{\prime} e^{-\varepsilon^{2}|\operatorname{Re} \zeta|} \quad(k=1,2, \cdots)
$$

for some constant $C^{\prime}$ independent of $k$. Thus we have 


$$
\left|J(\zeta) \cdot \varphi_{k}(\zeta)\right| \leqq \frac{C^{\prime}}{k} \cdot e^{-\varepsilon^{2} \mid \operatorname{Re} \zeta 1},
$$

and this implies $J \cdot \varphi_{k}$ converges to 0 in $\mathscr{Q}_{*}$. (q. e. d. for Theorem 3.2'.)

The idea of this proof was suggested by Prof. T. Oshima.

Proof of Theorem 3.3'. By Lemma 3.5 we find it sufficient to show that the multiplication operator

$$
J \cdot: \mathscr{P}_{*} \longrightarrow J \mathscr{P}_{*}
$$

is a linear topological isomorphism. We can show this from the following claim. (q.e.d. for Theorem 3.3'.)

Claim. Let $\delta$ be a positive conslant and $J$ an element of $G^{n}$ with the properties:

i) $J$ satisfies the condition ( $\left.\mathrm{S}^{\prime}\right)$,

ii) for any positive $\delta^{\prime}(<\delta)$, all the irreducible components of the zero variety of $J$ in $\left\{\zeta \in \boldsymbol{C}^{n} ;|\operatorname{Im} \zeta|<\delta^{\prime}\right\}$ intersect with $\boldsymbol{R}^{n}$.

For $a$ positive $\varepsilon$ with $6 \varepsilon<\delta$ and a real analytic function $\varphi(\zeta)$, we assume,

iii) $J \cdot \varphi$ extends holomorphically to the domain $D=\boldsymbol{R}^{n} \times \sqrt{-1} B_{R n}(0 ; 6 \varepsilon)$,

iv) $|J(\zeta) \cdot \varphi(\zeta)| \leqq e^{-6 \varepsilon \mid \operatorname{Re} \zeta 1}$ on $D$.

Then $\varphi$ is holomorphic in $D$ and there exists a constant $C$ independent of $\varphi$ for which $\varphi$ satisfies the estimate

$$
|\varphi| \leqq C e^{-\varepsilon|\operatorname{Re} \zeta|} \quad(|\operatorname{Im} \zeta|<\varepsilon) .
$$

Proof of Claim. The part about the domain of holomorphy of $\varphi$ is clear from the assumption ii). Since $J \in G^{n}$, there exists a positive number $C_{1}$ with the estimate

$$
|J(\zeta)| \leqq C_{1} e^{\varepsilon|\operatorname{Re} \zeta|} \quad(|\operatorname{Im} \zeta| \leqq 6 \varepsilon) .
$$

Since $J$ satisfies (S'), there exists a positive $N$ such that for any $\xi \in \mathbb{R}^{n}$ with $|\xi|>N$ we can take a point $\eta_{\xi} \in \mathbb{C}^{n}$ satisfying
a) $\left|\xi-\eta_{\xi}\right|<\varepsilon$,
b) $\left|J\left(\eta_{\xi}\right)\right| \geqq e^{-\varepsilon|\xi|}$.

Now we apply Lemma 3.7 to the pair $(J \cdot \varphi, J)$ on $B_{C n}\left(\eta_{\xi} ; 4 \varepsilon\right)$. On $B_{C n}\left(\eta_{\xi} ; 4 \varepsilon\right)$, we have the estimates

$$
\begin{aligned}
& |J \cdot \varphi| \leqq e^{-6 \varepsilon|\operatorname{Re} \zeta|}<e^{-6 \varepsilon(|\xi|-5 \varepsilon)}, \\
& |J| \leqq C_{1} e^{\varepsilon|\operatorname{Re} \zeta|}<C_{1} e^{\varepsilon(|\xi|+5 \varepsilon)} .
\end{aligned}
$$

Hence we have on $B_{C n}\left(\eta_{\xi} ; 2 \varepsilon\right)$ 


$$
\begin{aligned}
|\varphi| & =\left|\frac{J \cdot \varphi}{J}\right| \leqq e^{-6 \varepsilon|\xi|+30 \varepsilon^{2}} \cdot C_{1}^{2} e^{2 \varepsilon|\xi|+10 \varepsilon^{2}} \cdot e^{3 \varepsilon \mid \xi !} \\
& =C_{1}^{2} e^{40 \varepsilon^{2}} \cdot e^{-\varepsilon|\xi|} .
\end{aligned}
$$

We remark that $B_{C n}(\xi ; \varepsilon) \subset B_{C n}\left(\eta_{\xi} ; 2 \varepsilon\right)$, and obtain the estimates

$$
\begin{aligned}
|\varphi(\zeta)| & \leqq C_{1}^{2} e^{40 \varepsilon^{2}} \cdot e^{-\varepsilon|\xi|} \\
& \leqq C_{1}^{2} e^{40 \varepsilon^{2}} \cdot e^{-\varepsilon(|\operatorname{Re} \zeta|-\varepsilon)} \\
& \leqq C_{1}^{2} e^{41 \varepsilon^{2}} \cdot e^{-\varepsilon \mid \operatorname{Re} \zeta ; \quad \text { on } B_{C n}(\xi ; \varepsilon) .}
\end{aligned}
$$

Put $C_{2}=C_{1}^{2} e^{41 \varepsilon^{2}}$. Then we have

$$
\mid \varphi(\zeta) i \leqq C_{2} e^{-\varepsilon|\operatorname{Re} \zeta|}
$$

on the domain $\left\{\zeta \in C^{n} ;|\operatorname{Re} \zeta|>N,|\operatorname{Im} \zeta|<\delta\right\}$. We must estimate $|\varphi(\zeta)|$ on $\left\{\zeta \in C^{n} ;|\operatorname{Re} \zeta| \leqq N,|\operatorname{Im} \zeta|<\delta\right\}$. We set

$$
\tilde{J}(\xi)=\max _{|\eta-\xi| \leqq \varepsilon}|J(\eta)| \quad \text { for } \xi \in \boldsymbol{R}^{n},|\xi| \leqq N .
$$

Since $\tilde{J}(\xi)>0$ and $\left\{\xi \in \boldsymbol{R}^{n} ;|\xi| \leqq N\right\}$ is compact, we have

$$
\min _{\xi \xi 1 \leqq N} \tilde{J}(\xi)>0 \text {. }
$$

Then from Lemma 3.7 we can also deduce the estimate

$$
|\varphi(\zeta)| \leqq C_{3} \quad \text { on }\left\{\zeta \in C^{n} ;|\operatorname{Re} \zeta| \leqq N,|\operatorname{Im} \zeta|<\delta\right\}
$$

for some constant $C_{3}$ independent of $\varphi$. Put $C=\max \left\{C_{2}, C_{3} e^{\varepsilon N}\right\}$. We obtain the estimate

$$
|\varphi(\zeta)| \leqq C e^{-\varepsilon|\operatorname{Re} \zeta|} \quad \text { on }\left\{\zeta \in C^{n} ;|\operatorname{Im} \zeta|<\varepsilon\right\},
$$

which is the desired result. (q.e.d. for Claim.)

Proof of Theorem 3.4'.

For i): We put

$$
J(\zeta)=\prod_{k=1}^{\infty}\left(1-\frac{\zeta}{3^{k}+\sqrt{-1 / k}}\right) .
$$

On account of Lemma 3.8, $J$ belongs to $G_{0}^{1}$, and $\left\{3^{k}+\sqrt{-1} / k\right\}_{k \in N}$ are the zeros of $J$. Then we find out that $J$ does not satisfy (Z'). We will show that $J$ satisfies ( $\mathrm{S}^{\prime}$ ). It is sufficient to estimate $J(\zeta)$ when $\operatorname{Re} \zeta \gg 0$. For $\xi>1$, we take $N \in \boldsymbol{N}$ with $3^{N-1} \leqq \xi<3^{N}$. Then

$$
\begin{aligned}
\log |J(\xi)| & =\sum_{k=1}^{\infty} \log \left|1-\frac{\xi}{3^{k}+\sqrt{-1 / k}}\right| \\
& =\sum_{k=1}^{N-2}+\sum_{k=N-1}^{N}+\sum_{k=N+1}^{\infty} \cdot
\end{aligned}
$$


The first term is non-negative and the third term is estimated by

$$
\sum_{k=N+1}^{\infty} \log \left|1-\frac{\xi}{3^{k}+\sqrt{-1 / k}}\right| \geqq \sum_{j=1}^{\infty} \log \left(1-\frac{1}{3^{j}}\right) .
$$

Then the third term is bounded from below by some constant $C$ independent of $\xi$ and $N$. The second term is estimated by

$$
\begin{aligned}
\log \left|1-\frac{\xi}{3^{N-1}+\frac{\sqrt{-1}}{N-1} \mid}\right| & +\log \left|1-\frac{\xi}{3^{N}+\sqrt{-1 / N}}\right| \\
& \geqq 2 \log \frac{1}{N} \cdot \frac{1}{3^{N}+1}>2 \log 3^{-2 N} \\
& \geqq-4 \log 3 \xi .
\end{aligned}
$$

After all we can take some constants $C_{1}$ and $C_{2}$ such that

$$
\log |J(\xi)| \geqq-C_{1} \log \xi-C_{2} \quad(\xi>1) .
$$

This implies the desired result.

For ii): We will construct $J$ with desired properties in case $n=1$. If we regard $J$ as a function of $n$ variables, $J$ also enjoys desired properties in the general case. We take $\zeta_{k} \in \boldsymbol{C}$ for $k \in \boldsymbol{N}$ with

$$
\begin{aligned}
\text { i) } & \left|\zeta_{k}\right|=k^{2} \cdot 3^{k^{2}}, \\
\text { ii) } & \operatorname{Re} \zeta_{k}>0 \\
\text { iii) } & \operatorname{Im} \zeta_{k}=\frac{k^{2}}{3},
\end{aligned}
$$

and put

$$
J(\zeta)=\prod_{k=2}^{\infty}\left(1-\frac{\zeta}{\zeta_{k}}\right)^{3^{2}}
$$

If we remark the above condition iii), we can prove the following claim which shows the desired properties. (q.e.d. for Theorem 3.4'.)

Claim. $J$ becomes an entire function and has the properties

a) for any $\varepsilon>0$, there exists a positive constant $C_{\varepsilon}$ such that

$$
|J(\zeta)| \leqq C_{\varepsilon} e^{\varepsilon|\zeta|},
$$

b) $J$ has no zeros in the domain $\{\zeta \in C ;|\operatorname{Im} \zeta|<1\}$,

c) we can take positive constants $\delta$ and $K$ such that

$$
|J(\zeta)| \leqq e^{-\delta \zeta_{k} \mid} \quad\left(\left|\zeta-\zeta_{k}\right|<k^{2}, k>K\right) .
$$

Proof of Claim. a) and b) are clear from Lemma 3.8 and the estimate 


$$
\sum_{k=2}^{\infty} \frac{1}{k^{2} \cdot 3^{k^{2}}} \times 3^{k^{2}}=\sum_{k=2}^{\infty} \frac{1}{k^{2}}<+\infty .
$$

To prove $\mathrm{c}$ ), first remark the estimate with a fixed $k$ :

$$
\begin{aligned}
\log |J(\zeta)| & =\sum_{j=2}^{\infty} 3^{j^{2}} \log \left|1-\frac{\zeta}{\zeta_{j}}\right| \\
& \leqq 3^{k^{2}} \log \left|1-\frac{\zeta}{\zeta_{k}}\right|+\sum_{j=2}^{\infty} 3^{j^{2}} \log \left(1+\left|\frac{\zeta}{\zeta_{j}}\right|\right) .
\end{aligned}
$$

The second term is estimated as

$$
\begin{aligned}
\sum_{j=2}^{\infty} 3^{j^{2}} \log \left(1+\frac{|\zeta|}{j^{2} 3^{j^{2}}}\right) & \leqq \sum_{j=2}^{\infty} 3^{j^{2}} \cdot \frac{|\zeta|}{j^{2} 3^{j^{2}}}=|\zeta| \sum_{j=2}^{\infty} \frac{1}{j^{2}} \\
& \leqq|\zeta| \sum_{j=2}^{\infty} \frac{1}{j(j-1)}=|\zeta|<\left(1+3^{-k^{2}}\right)\left|\zeta_{k}\right|,
\end{aligned}
$$

if $\left|\zeta-\zeta_{k}\right|<k^{2}$. The first term is estimated as

$$
3^{k^{2}} \cdot \log \left|\frac{\zeta-\zeta_{k}}{\zeta_{k}}\right| \leqq 3^{k^{2}} \cdot \log \frac{k^{2}}{k^{2} \cdot 3^{k^{2}}}=-\left|\zeta_{k}\right| \cdot \log 3,
$$

if $\left|\zeta-\zeta_{k}\right|<k^{2}$. Hence we have on $B_{C}\left(\zeta_{k} ; k^{2}\right)$ the estimate

$$
\begin{aligned}
\log |J(\zeta)| & <-\left|\zeta_{k}\right|\left\{\log 3-\left(1+3^{-k^{2}}\right)\right\} \\
& =-\left|\zeta_{k}\right|\left(\log \frac{3}{e}-3^{-k^{2}}\right) .
\end{aligned}
$$

In this situation, it suffices to take $\delta$ and $K$ so that

$$
0<\delta<\log \frac{3}{e}-3^{-K^{2}}
$$

(q. e. d. for Claim.)

This example in Theorem 3.4 ii) is due to Prof. T. Oshima.

\section{References}

[Be] Beuling, A., Quasi-analyticity and general distributions, Lecture 4 and 5. A.M.S. Summer institute, Stanford, 1961 (mimeographed).

[Bj] Björk, G., Linear partial differential operators and generalized distributions, Ark. Mat., 6 (1966), 351-407.

[Bo] Boas, R.P., Entire Functions, New York, 1954.

[E-1] Ehrenpreis, L., Solutions of some problems of division IV, Amer. J. Math., 82 (1960), 522-588.

[E-2] - Fourier analysis in several complex variables, Wiley-Interscience, New York, 1970.

[G] Grothendieck, A., Sur les espaces (T) et (DIF), Summa Brazil. Math., 3 (1954), 57-122.

[H] Harvey, R., Hyperfunctions and partial differential equations, Thesis, Stanford 
Univ., 1966; its summary is given in Proc. Nat. Acad. Sci. U.S.A., 55 (1966), 1042-1046.

[Ka-1] Kawai, T., On the theory of Fourier hyperfunctions and its applications to partial differential equations with constant coefficients, J. Fac. Sci. Univ. Tokyo, Sec. IA, 17 (1970), 467-517.

[Ka-2] - The theory of Fourier transforms in the theory of hyperfunctions and its applications, Master's thesis presented to Univ. of Tokyo, 1970.

[Ko-1] Komatsu, H., Projective and injective limits of weakly compact sequences of locally convex spaces, J. Math. Soc. Japan., 19 (1967), 366-383.

[Ko-2] - Ultradistributions, I, Structure theorems and a characterization, J. Fac. Sci. Univ. Tokyo, Sec. IA, 20 (1973), 25-105.

[Ko-3] - Ultradistributions, II, The kernel theorem and ultradistributions with support in a submanifold, J. Fac. Sci. Univ. Tokyo, Sec. IA, 24 (1977), 607-628.

[L] Levin, B., Distributions of zeros of entire functions, Amer. Math. Soc. Transl. Providence, 1964.

[M] Martineau, A., non-published, see Théorème 1 in Schapira, P., Équations aux dérivées partielles dans l'espace des hyperfonctions, Lect. Notes in Math., Springer, 71 (1968), 38-45.

[Ra] Raikov, D. A., On two classes of locally convex spaces important in applications, Trudy Sem. Funkt. Anal. Voronež., 5 (1957), 27-34 (in Russian).

[Ro-1] Roumieu, C., Sur quelques extentions de la notion de distribution, Ann. Sci. Ecole Norm. Sup. Paris, 3 Sér, 77 (1960), 41-121.

[Ro-2] - Ultra-distributions définies sur $\boldsymbol{R}^{n}$ et sur certaines classes de variétés différentiables, J. Anal. Math., 10 (1962-63), 153-192.

[S] Sebastiaõ J.. e Silva, Su certe classi di spazi localmente convessi importanti per le applicazioni, Rend. Mat. Univ. Roma, Ser. 5, 15 (1955), 388-410.

[Y] Yoshinaga, K., On a locally convex space introduced by J.S.E. Silva, J. Sci. Hiroshima Univ., Ser. A, 21 (1957), 89-98. 